\title{
Sagnac Interference in Carbon Nanotube Loops
}

\author{
Gil Refael, ${ }^{1}$ Jinseong Heo, ${ }^{2}$ and Marc Bockrath ${ }^{2}$ \\ ${ }^{1}$ Department of Physics, California Institute of Technology, MC 114-36, Pasadena, California 91125, USA \\ ${ }^{2}$ Department of Applied Physics, California Institute of Technology, MC 114-36, Pasadena, California 91125, USA
}

(Received 22 August 2006; published 15 June 2007)

In this Letter we study electron interference in nanotube loops. The conductance as a function of the applied voltage is shown to oscillate due to interference between electron beams traversing the loop in two opposite directions, with slightly different velocities. The period of these oscillations with respect to the gate voltage, as well as the temperatures required for the effect to appear, are shown to be much larger than those of the related Fabry-Perot interference. We calculate interaction effects on the period of the oscillations, and show that even though interactions destroy much of the near degeneracy of velocities in the symmetric spin channel, the slow interference effects survive.

DOI: 10.1103/PhysRevLett.98.246803

PACS numbers: 73.63.Fg, 73.23.-b

Single-walled carbon nanotubes are a prime example of quantum strongly correlated phenomena in mesoscopic physics [1]. Luttinger-liquid behavior [2,3], as well as Fabry-Perot interference [4-6] were observed in these systems. The latter should disclose the interaction parameters of the Luttinger liquid. But since interference patterns of spin and charge modes have similar energy scales, such experimental observation is challenging. Indeed, the development of electron interferometery is crucial for probing interaction effects. Mach-Zehnder interferometers reveal Aharonov-Bohm oscillations and magentic-field edge channels, [7-11], and can probe exotic fractional quantum Hall states [12-14]. Two-path interferometers allow probing correlated states of quantum dots $[15,16]$. Universal conductance fluctuations (UCF) [17] and weak localization effects $[18,19]$, were measured in a controlled fashion using interference in ballistic quantum dots [20]. Similarly, interference is successfully used to probe electronic interactions in nanowires [21,22].

In this Letter, we analyze a new electronic interference mode in nanotubes - the Sagnac interference [23]. The optical Sagnac effect measures the angular velocity of a rotating ring, through interference between counterpropagating light beams. In an armchair nanotube loop [Fig. 1(a)], the same effect arises due to velocity detuning between right- and left-moving electrons, occurring when the electronic Fermi surface is tuned away from the Dirac nodes. This effect also reflects the interference underlying weak localization (the velocity detuning replaces the applied normal magnetic field); it similarly produces largeperiod conductance fluctuations as a function of gate- and source-drain voltages, and survives to very high temperatures (unlike UCF which is sensitive to thermal dephasing). Figure 1(b) shows, we believe, such a large-period fluctuation, which appears already at $T=64 \mathrm{~K}$. Following the discussion of the theory of Sagnac interference in nanotubes, we will address this data, considering also FabryPerot interference in this geometry.
The Sagnac interference in armchair nanotubes appears when the Fermi surface is shifted from the band middle using a gate voltage, $V_{g}$. Ignoring interactions, this detunes the velocities of right and left-moving electrons in each node: $v_{R}=v_{F}+u$, and $v_{L}=v_{F}-u$ [Fig. 2(a)] [24].

a.

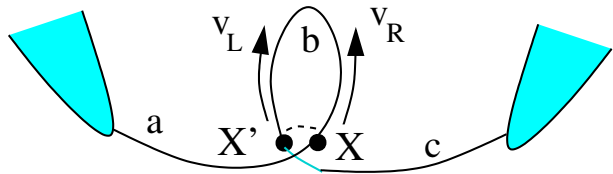

b.

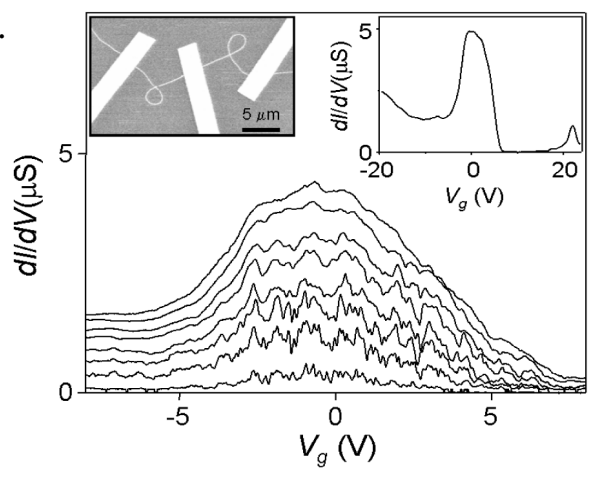

FIG. 1 (color online). (a) In a nanotube loop, electrons can tunnel (dashed line) from one branch $(X)$ to the other $\left(X^{\prime}\right)$ [33,34]. Electrons impinging on point $X$ either proceed through the loop moving counterclockwise, or tunnel to $X^{\prime}$, and proceed clockwise. Velocity difference between right and left movers produces Sagnac interference between the counterpropagating beams. (b) Conductance vs gate voltage of a nanoloop device. From top to bottom: $T=64,48,32,24,16,12,8$, and $4 \mathrm{~K}$. Strong conductance fluctuations, consistent with Sagnac interference, appear already at $T=64 \mathrm{~K}$ with period $\delta V_{G} \sim 20 \mathrm{~V}$. At lower temperatures Fabry-Perot interference appears as well with short periods $\delta V_{g} \sim 0.15 \mathrm{~V}, 0.3 \mathrm{~V}$. Left inset shows the device; data from both loops are qualitatively the same. Right inset shows the suspected Sagnac envelope at $T=4 \mathrm{~K}$ and $V_{\text {sd }}=30 \mathrm{mV}$ for wider range of $V_{g}$. Note that the asymmetry in $V_{g}$ is not understood. 
The small difference $u$, produces a slow fluctuation of the conductance as a function of $V_{g}$ [25] through a phase difference between the counterpropagating beams:

$$
\Delta \phi=L k_{L}-L k_{R}=\frac{L \epsilon_{F}}{\hbar v_{L}}-\frac{L \epsilon_{F}}{\hbar v_{R}} \approx \alpha e V_{g} L \frac{2 u}{\hbar v_{F}^{2}},
$$

where $L$ is the length of the loop, and $\epsilon_{F}=\hbar v_{R / L} k_{R / L}$. Also, $\epsilon_{F}=\alpha e V_{g}$, where $\alpha$ is the conversion factor between the gate voltage and change in chemical potential; $\alpha$ is estimated to be of the order of $10^{-2}$ in the system of Fig. 1(b) [27]. Interference fringes repeat when $\Delta \phi=$ $2 \pi n$. Since roughly $u \propto V_{g}$, the $n$th fringe is at $V_{g} \propto \sqrt{n}$; fringes are more dense as we move away from the middle of the nanotube's band. For noninteracting electrons, the same fringes should appear as a function of a source-drain voltage, $V_{\text {sd }}$. In the armchair-tube nanoloop, beams moving in the same direction around the loop, but in different nodes, also interfere [Fig. 2(c)]. The two beams in this band-Sagnac effect differ by the same phase due to the time-reversal symmetry.

The above picture completely ignores interactions. But the thin single-walled nanotubes we probed probably have a Luttinger parameter $g \sim 0.3$, and are not a Fermi-liquid [4]. Interactions change the hydrodynamic velocities in the nanotube dramatically, and may lift the near velocity degeneracy of interfering beams. Next, we analyze the Sagnac interference (and the Fabry-Perot with $u \neq 0$ ) of interacting electrons. We show that interactions do not destroy the large-period Sagnac fringes. The fringes in the conductance vs $V_{g}$ are determined mostly by the bare, noninteracting, velocity spectrum, essentially reflecting Eq. (1). Nevertheless, the fringes in $V_{\text {sd }}$ are modified dramatically: they reflect the four velocities of the tube's hydrodynamic modes.

The bosonized Lagrangian of the two Dirac nodes, with $\lambda$ parametrizing the density-density interaction, is

$$
\begin{aligned}
\mathcal{L}= & \frac{\hbar v_{F}}{2 \pi} \sum_{\sigma, a=1,2} \int d x\left[\frac{2}{v_{F}} \dot{\theta}_{a}^{\sigma} \nabla \phi_{a}^{\sigma}-\left(\nabla \theta_{a}^{\sigma}\right)^{2}-\left(\nabla \phi_{a}^{\sigma}\right)^{2}\right. \\
& \left.+(-1)^{a} 2 \frac{u}{v_{F}} \nabla \phi_{a}^{\sigma} \nabla \theta_{a}^{\sigma}\right]-\int d x \lambda\left(\sum_{\sigma, a=1,2} \frac{1}{\pi} \nabla \theta_{a}^{\sigma}\right)^{2} .
\end{aligned}
$$

The subscript $a$ designates the Dirac node, $\sigma$ is the spin. The charge mode, $\frac{1}{2} \sum_{\sigma, a=1,2}\left(\theta_{a}^{\sigma}\right)$, has the interaction Luttinger parameter $g=\left(1+8 \lambda / \pi \hbar v_{F}\right)^{-1 / 2}$. The Fermionic operators are: $\psi_{a R / L}^{\sigma} \sim e^{i(-1)^{a}\left(2 \pi / 3 a_{0}\right) x-i\left(\phi_{a}^{\sigma} \pm \theta_{a}^{\sigma}\right)}$.

The Fourier-transform of (2) defines an $8 \times 8$ quadratic form of the $\theta$ 's and $\phi$ 's. Its eight eigenvalues are squareroots of second degree polynomials of $\omega$ and $k$. The dispersion of the eight chiral modes is given by the values of $\omega / k$ which make an eigenvalue vanish. We find that the spin antisymmetric channel consists of four untouched neutral chiral modes, with velocities: $v_{1+}^{\perp}= \pm v_{F}+u$, $v_{2 \pm}^{\perp}= \pm v_{F}-u$, where + and - indicate right and left
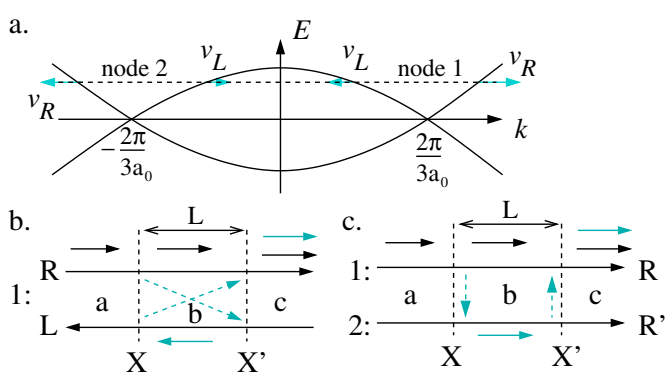

FIG. 2 (color online). (a) Dispersion of an armchair nanotube. When the Fermi surface is away from the nodes, right and left movers in each node have different velocities, $v_{R / L}=v_{F} \pm u$ ( $a_{0}$ is the lattice constant), which leads to two Sagnac interference effects: (b) Within one node (say node 1), a beam entering the loop from the left (short black arrow) splits by partially tunneling between points $X$ and $X^{\prime}$ to two counterpropagating beams (black and gray), in region $b$. They then recombine at point $X^{\prime}$. Long black arrows represent the two chiral electronic modes near node 1 . The regions $a, b$, and $c$ correspond to those indicated in Fig. 1. (c) A beam impinging on point $X$ in node 1 (2) partially scatters to node 2 (1). Both traverse the loop in the same direction (short black and gray arrows), and recombine at point $X^{\prime}$. This effect is similar to the slow conductance oscillation due to impurities propounded in Ref. [35], and in the presence of axial magnetic field in Ref. [36].

movers, respectively. More interestingly, the remaining four spin-symmetric chiral modes are given by (see also Ref. [28]):

$$
\frac{v_{1,2}^{\|}}{v_{F}}=\frac{1}{\sqrt{2}} \sqrt{1+\frac{1}{g^{2}}+2 \frac{u^{2}}{v_{F}^{2}} \pm \sqrt{\left(1-\frac{1}{g^{2}}\right)^{2}+8 \frac{u^{2}}{v_{F}^{2}}\left(1+\frac{1}{g^{2}}\right)},}
$$

$v_{1}^{\|}$and $v_{2}^{\|}$describe two right-left symmetric branches of the spectrum. $v_{1}^{\|}$is the charge mode velocity, when $u=0$. Next, we determine which velocities appear in the interference fringes of $V_{g}$ and $V_{\text {sd }}$.

$V_{g}$ couples to the total electronic density, whereas $V_{\text {sd }}$ couples to the density difference of right and left movers:

$$
\mathcal{L}_{g+\mathrm{sd}}=\int d x \sum_{\sigma, a}\left(\alpha e V_{g} \frac{1}{\pi} \nabla \theta_{a}^{\sigma}+e V_{\mathrm{sd}} \frac{1}{\pi} \nabla \phi_{a}^{\sigma}\right) .
$$

$V_{g}$ changes the chemical potential and Fermi surface of the electrons. This is seen by absorbing the new term in the $\theta$ and $\phi$ gradient terms (where the latter is involved only due to the difference in the original right and left moving velocities). Unlike $V_{g}, V_{\text {sd }}$ drives the system out of equilibrium: it induces a current. This entails a time-dependent transformation of the bosonic fields to absorb the term. The transformation

$$
\begin{aligned}
& \tilde{\theta}_{a}^{\sigma}=\theta_{a}^{\sigma}-\frac{\alpha g^{2} e V_{g}}{\hbar v_{F}} \frac{1}{1-g^{2} u^{2} / v_{F}^{2}} x+\frac{e V_{\mathrm{sd}}}{\hbar} t, \\
& \tilde{\phi}_{a}^{\sigma}=\phi_{a}^{\sigma}-(-1)^{a} \frac{\alpha g^{2} e V_{g}}{\hbar v_{F}} \frac{u / v_{F}}{1-g^{2} u^{2} / v_{F}^{2}} x,
\end{aligned}
$$


absorbs both $V_{g}$ and $V_{\text {sd }}$ in the bosonic fields, with $\sigma=\uparrow$, $\downarrow$, and $a=1,2$ is the node. The slow, $u$-dependent fluctuations can already be noticed in $\phi_{a}^{\sigma}$ 's $x$ dependence. The above procedure is drawn from Ref. [5].

Following are the possible scattering processes contributing to transport through the nanoloop. For simplicity, we define $\tilde{\psi}_{a R / L}^{\sigma} \sim e^{i(-1)^{a}\left(2 \pi / 3 a_{0}\right) x-i\left(\tilde{\phi}_{a}^{\sigma} \pm \tilde{\theta}_{a}^{\sigma}\right)}$. The simplest term is the same-node backscattering (in the following we omit the Hermitian conjugates):

$$
\hat{B}(x, t)=b \sum_{\sigma, a}\left(\tilde{\psi}_{a}^{\sigma \dagger}(x) \tilde{\psi}_{a}^{\sigma}(x) e^{2 i k_{g} x-2 i \omega_{\mathrm{sd}} t}\right),
$$

$$
\text { with } k_{g}=\frac{\alpha e g^{2} V_{G}}{\hbar v_{F}\left(1-g^{2} u^{2} / v_{F}^{2}\right)}, \quad \omega_{\mathrm{sd}}=e V_{\mathrm{sd}} / \hbar v_{F} .
$$

Second is cross-node backscattering: $\hat{N}_{b}(x, t)=$

$$
n_{b} \sum_{\sigma, a}\left(\tilde{\psi}_{a}^{\sigma \dagger}(x) \tilde{\psi}_{\bar{a}}^{\sigma}(x) e^{2 i k_{g}\left(1+(-1)^{a}\left(u / v_{F}\right)\right) x-2 i \omega_{\mathrm{sd}} \mathrm{t}}\right),
$$

which is a backscattering from node $a$, to node, $\bar{a}=3-a$. Third is cross-node forwardscattering: $\hat{N}_{f}(x, t)=$

$$
n_{f} \sum_{\sigma, a}\left(\tilde{\psi}_{a}^{\sigma \dagger}(x) \tilde{\psi}_{\bar{a}}^{\sigma}(x)+\tilde{\psi}_{a}^{\sigma \dagger}(x) \tilde{\psi}_{\bar{a}}^{\sigma}(x)\right) e^{2 i(-1)^{a}\left(k_{g} u / v_{F}\right) x} .
$$

Most important is the backscattering term arising from tunneling between point $X$ at $x=0$, and $X^{\prime}$ at $x=L$ [Fig. 2(a)], i.e., cross-loop backscattering:

$$
\begin{aligned}
\hat{K}_{b}(t)= & k_{b} \sum_{\sigma, a, b}\left(\tilde{\psi}_{a}^{\sigma \dagger}(0) \tilde{\psi}_{b}^{\sigma}(L) e^{i k_{g}\left(1-(-1)^{b}\left(u / v_{F}\right)\right) L-2 i \omega_{\mathrm{sd}} t}\right. \\
& \left.+\tilde{\psi}_{a}^{\sigma \dagger}(0) \tilde{\psi}_{b}^{\sigma}(L) e^{-i k_{g}\left(1+(-1)^{b}\left(u / v_{F}\right)\right) L+2 i \omega_{\mathrm{sd}} t}\right) .
\end{aligned}
$$

To calculate the conductance fluctuations, we must follow the Kubo-Keldysh formalism as it applies to the various scattering events, $\hat{L}_{m}(x, t)$, where $\hat{L}_{m}=\hat{B}, \hat{N}_{f / b}, \hat{K}_{b}$. We defer an exact evaluation to a later publication, and concentrate here on the main features of the fluctuations:

$$
\Delta G_{m n} \sim \int_{0}^{\infty} d t\left\langle\left[\hat{L}_{m}(t), \hat{L}_{n}(0)\right]\right\rangle \sim\left\langle\hat{L}_{m} \hat{L}_{n}\right\rangle_{\omega=2 e V_{\mathrm{sd}} / \hbar} .
$$

The second relation, connecting the integral to the correlation's Fourier transform, is due to the time dependence of the integrand being $e^{2 i \omega_{\mathrm{sd}} t}=e^{2 i e V_{\mathrm{sd}} t / \hbar}$. In the case of $\hat{L}_{m}$ occurring at $x=0$ and $\hat{L}_{m}$ at $x=L$, the dependence of the oscillating part of $\Delta G_{m n}$ on $V_{\mathrm{sd}}$ is easily seen to be of the form

$$
\Delta G_{m n}=f\left(e^{2 i \omega_{\mathrm{sd}} L / v_{1}^{\|}}, e^{2 i \omega_{\mathrm{sd}} L / v_{2}^{\|}}, e^{2 i \omega_{\mathrm{sd}} L / v_{1+}^{\perp}}, e^{2 i \omega_{\mathrm{sd}} L / v_{2+}^{\perp}}\right),
$$

where $v_{1 / 2}^{I / / \perp}$ are the four velocities of the hydrodynamic modes described in and above Eqs. (3). Thus the conductance vs $V_{\text {sd }}$ fringes are determined by the velocities of the interaction-induced four hydrodynamic modes.

Focusing on conductance $G$ vs $V_{g}$, we see that only $\exp \left(2 i k_{g} L\right)$ and $\exp \left(2 i k_{g} L \frac{u}{v_{F}}\right)$ produce interference effects in $V_{g}\left[k_{g}\right.$ defined in Eq. (7)]. Table I lists the conductance fluctuations due to second-order scattering. Our focus, loop-Sagnac interference of counterpropagating beams, is given in the first line in Table I). Indeed, it coincides with the band-Sagnac interference (B-SAG); it is possible, however, to distinguish the two Sagnac modes by applying a magnetic flux to the loop. The band-Sagnac fringes will be unaffected, whereas the loop-Sagnac amplitude would be suppressed to first order by $\cos (2 e \Phi / \hbar)$, with $\Phi$ the flux through the loop.

The temperature sensitivity of the Sagnac and FabryPerot interference differ remarkably. Let us estimate and compare the two. An electron has energy uncertainty $\sim T$, tantamount to an uncertainty in $V_{g}: \Delta V_{g} \sim T_{c} / \alpha g^{2} e$. Comparing $\Delta V_{g} \approx \delta V_{g}$, where the interference disappears, yields $T_{c}$ (Table I). Note that the $g^{2}$ factor is abscent in the $T_{c}$ expressions; since temperature only smears the kinetic energy of electrons, the effects of interactions should be omitted to first approximation from $T_{c}$. We find that $T_{c}$ for the Sagnac modes is $v_{F} / u$ larger than that of the Fabry-Perot interference.

$$
T_{c}^{\mathrm{SAG}} \sim \frac{\pi \hbar v_{F}}{L}\left(1-\frac{u^{2} g^{2}}{v_{F}}\right) \frac{v_{F}}{u} \sim \frac{v_{F}}{u} T_{c}^{\mathrm{FP}} .
$$

Hence the limiting factor for the observation of the Sagnac effect is most likely phonon scattering.

The measurement motivating this work is shown in Fig. 1. At $T=32 \mathrm{~K}$ fast oscillations with period $\delta V_{g} \sim$ $0.3 \mathrm{~V}$ appear; we identify them as the loop-FP mode of Table I (Coulomb blockade depends on total wire length, and is expected at a much lower $\delta V_{g} \sim 10^{-3}-10^{-2} \mathrm{~V}$ ). Another fast mode appears at $T=12 \mathrm{~K}$, with a doubled frequency, $\delta V_{g} \sim 0.15 \mathrm{~V}$, fitting the regular FP mode. In addition, a slowly oscillating envelope is already evident at $T=64 \mathrm{~K}$, with the first period being roughly $\delta V_{g} \sim 20 \mathrm{~V}$ [29]. If we identify this with the Sagnac effects, then

TABLE I. Interfering contributions to the conductance as a function of $V_{g}$. The first row indicates the type of interference. The loop-Sagnac (L-SAG) and band-Sagnac (B-SAG) correspond to Figs. 2(b) and 2(c) respectively. The three FabryPerot modes originate from electronic path difference: of twoloops in node 1 or 2 (B-FP \pm ), of one loop in node 1 and one in 2 (FP), or, due to loop-tunneling, one loop in node 1 or 2 (L-FP \pm ). The coherence temperature of each interference mode is determined heuristically by assuming that $T_{c} \sim \alpha g^{2} e \delta V_{g} / k_{B}$.

\begin{tabular}{cccc}
\hline \hline Type & $\hat{L}_{m} \hat{L}_{n}$ & $\delta V_{g}$ & $T_{c}$ \\
\hline L-SAG & $\hat{K}_{b} \hat{K}_{b}$ & $\frac{\pi \hbar v_{F}}{\alpha e g^{2} L}\left(1-\frac{u^{2} g^{2}}{v_{F}^{2}}\right) \frac{v_{F}}{u}$ & $\frac{\pi \hbar v_{F}}{L}\left(1-\frac{u^{2} g^{2}}{v_{F}^{2}}\right) \frac{v_{F}}{u}$ \\
B-SAG & $\hat{N}_{f}\left(\hat{N}_{b} \hat{B}\right)$ & $\frac{\pi \hbar v_{F}}{\alpha e g^{2} L}\left(1-\frac{u^{2} g^{2}}{v_{F}^{2}}\right) \frac{v_{F}}{u}$ & $\frac{\pi \hbar v_{F}}{L}\left(1-\frac{u^{2} g^{2}}{v_{F}^{2}}\right) \frac{v_{F}}{u}$ \\
FP & $\hat{B} \hat{B}$ & $\frac{\pi \hbar v_{F}}{\alpha e g^{2} L}\left(1-\frac{u^{2} g^{2}}{v_{F}^{2}}\right)$ & $\frac{\pi \hbar v_{F}}{L}\left(1-\frac{u^{2} g^{2}}{v_{F}^{2}}\right)$ \\
B-FP \pm & $\hat{N}_{b} \hat{N}_{b}$ & $\frac{\pi \hbar v_{F}^{2}}{\alpha e g^{2} L} \frac{1-u^{2} g^{2} / v_{F}^{2}}{v_{F} \pm u}$ & $\frac{\pi \hbar v_{F}^{2}}{L} \frac{1-u^{2} g^{2} / v_{F}^{2}}{v_{F} \pm u}$ \\
L-FP \pm & $\hat{K}_{b} \hat{B}, \hat{K}_{b} \hat{N}_{b}$ & $\frac{2 \pi \hbar v_{F}^{2}}{\alpha e g^{2} L} \frac{1-u^{2} g^{2} / v_{F}^{2}}{v_{F} \pm u}$ & $\frac{2 \pi \hbar v_{F}^{2}}{L} \frac{1-u^{2} g^{2} / v_{F}^{2}}{v_{F} \pm u}$ \\
\hline \hline
\end{tabular}


$\delta V_{g}^{\mathrm{SAG}} / \delta V_{g}^{\mathrm{FP}} \sim 130$. To check feasibility, we approximate the armchair nanotube's dispersion as parabolic, $\pm \epsilon_{k}=$ $-\gamma\left(1-\left(\frac{2 \pi}{3 a_{0}}\right)^{2} k^{2}\right) ;$ and $\frac{u}{v_{F}} \approx \frac{\epsilon}{2 \gamma}[30]$. The first fringe due to the Sagnac interference appears when the accumulated phase difference between the two interfering beams is $\pi$ :

$$
\Delta \phi=\int_{0}^{\mu^{\mathrm{SAG}}} d \epsilon \frac{L}{\hbar v_{F}} \frac{2 u}{v_{F}} \approx \frac{L}{\hbar v_{F}} \frac{\left(\mu^{\mathrm{SAG}}\right)^{2}}{2 \gamma}=\pi,
$$

where the integral is necessary due to the dependence of $u / v_{F}$ on $\epsilon$. The first fringe due to Fabry-Perot interference is when $\mu^{F P} 2 L / \hbar v_{F}=\pi$. Using $L=7 \mu \mathrm{m}$ and $v_{F}=$ $8 \times 10^{5} \mathrm{~m} / \mathrm{s}$, and $\gamma \approx 2.5 \mathrm{eV}$ [24], we obtain $V_{g}^{\mathrm{SAG}} / V_{g}^{\mathrm{FP}}=\mu^{\mathrm{SAG}} / \mu^{\mathrm{FP}} \sim 300$, which agrees with the experiment up to a factor of 2 . This extra factor might be due to the Fabry-Perot interference arising not from the loop, but from the shorter sections of the nanotube. The experimental results are also consistent with the fact that the Fabry-Perot interference is expected roughly at $T_{c}^{\mathrm{FP}} \sim$ $\frac{2 \pi \hbar v_{F}}{L} \sim 10 \mathrm{~K}$. Since currently only two samples of the loop geometry are available, we limit ourselves to the order-of-magnitude analysis above, and defer a detailed analysis of the experiment to a future publication.

In this Letter we discussed the Sagnac and Fabry-Perot interference in interacting nanotube loops, with $v_{R}-$ $v_{L}=2 u \neq 0$. We found that $V_{g}$ changes the "carrier wave" and induces fluctuations that depend mostly on the bare dispersion of the nanotube, while $V_{\text {sd }}$ produces fluctuations whose periods depend on the velocities of the nonequilibrium hydrodynamic modes. From these conductance fluctuations, one could in principle extract all hydrodynamic velocities, the interaction parameter, and the bare electron dispersion. We also provided rough estimates of the coherence temperatures, $T_{c}$, required to see the Sagnac interference, and showed that it is much higher than that of the Fabry-Perot interference. In fact, phonon scattering is most likely the limiting factor for the Sagnac effect at high temperatures. The estimates of $T_{c}$ are expected to be modified by a precise inclusion of interactions; this we will pursue in a future publication, in addition to the explicit dependence of $G$ on $V_{\text {sd }}$. As mentioned above, the Sagnac interference is closely related to the origin of weak localization; therefore, its analysis could directly determine the temperature and interaction dependence of the electronic dephasing time $\tau_{\phi}$ (see further in Refs. [31,32]). Here we showed that the Sagnac effect clearly survives interactions at $T=0$; therefore, our results can be interpreted as evidence for the divergence of $\tau_{\phi}$ in an interacting non-Fermi-liquid electronic system.

We are grateful to J. von Delft and Y. Oreg for a critical discussion, and to L. Balents, E. Demler, D. Feldman, V. Galitski, and J. Meyer for helpful comments. This research was supported in part by the National Science Foundation under Grant No. PHY05-51164. M. B. and J. H. are grateful to support by the ONR.
[1] S. J. Tans, M.H. Devoret, R. J.A. Groeneveld, and C. Dekker, Nature (London) 394, 761 (1998).

[2] C. Kane, L. Balents, and M. P. A. Fisher, Phys. Rev. Lett. 79, 5086 (1997).

[3] M. Bockrath et al., Nature (London) 397, 598 (1999).

[4] W. Liang et al., Nature (London) 411, 665 (2001).

[5] C. S. L. Balents and K. J. Wiese, Phys. Rev. B 68, 205423 (2003).

[6] J. Kong et al., Phys. Rev. Lett. 87, 106801 (2001).

[7] B. J. van Wees et al., Phys. Rev. Lett. 62, 2523 (1989).

[8] E. Buks et al., Nature (London) 391, 871 (1998).

[9] van der Wiel et al., Phys. Rev. B 67, 033307 (2003).

[10] H. Förster, S. Pilgram, and M. Büttiker, Phys. Rev. B 72, 075301 (2005).

[11] F. E. Camino, W. Zhou, and V. J. Goldman, Phys. Rev. B 72, 155313 (2005).

[12] D. E. Feldman and A. Kitaev, Phys. Rev. Lett. 97, 186803 (2006).

[13] P. Bonderson, A. Kitaev, and K. Shtengel, Phys. Rev. Lett. 96, 016803 (2006).

[14] A. Stern and B. I. Halperin, Phys. Rev. Lett. 96, 016802 (2006).

[15] A. Yacoby, M. Heiblum, D. Mahalu, and H. Shtrikman, Phys. Rev. Lett. 74, 4047 (1995).

[16] M. Sigristand et al., Phys. Rev. Lett. 98, 036805 (2007).

[17] P. A. Lee and A. D. Stone, Phys. Rev. Lett. 55, 1622 (1985).

[18] E. Abrahams et al., Phys. Rev. Lett. 42, 673 (1979).

[19] L. P. Gor'kov, D. Khmel'nitzkii, and A. I. Larkin, Pis'ma Zh. Eksp. Teor. Fiz. 30, 248 (1979).

[20] C. M. Marcus et al., Phys. Rev. Lett. 69, 506 (1992).

[21] O. M. Auslaender et al., Science 295, 825 (2002).

[22] M. Kindermann, P. W. Brouwer, and A. J. Millis, Phys. Rev. Lett. 97, 036809 (2006).

[23] G. Sagnac, C.R. des Academie des Sciences (Paris) 157, 708 (1913).

[24] M. Ouyang, J.-L. Huang, and C. M. Lieber, Phys. Rev. Lett. 88, 066804 (2002).

[25] Another interesting work that relies on $u \neq 0$ is Ref. [26].

[26] D. E. Feldman, Phys. Rev. Lett. 95, 177201 (2005).

[27] Consistent with previous experiments with back-gate geometry, and somewhat higher than $\alpha \sim 10^{-3}-10^{-2}$ in Meunier et al., Phys. Rev. Lett. 93, 246801 (2004).

[28] Y. Oreg and A. M. Finkel'stein, Phys. Rev. Lett. 74, 3668 (1995).

[29] Another fainter fluctuation with period $\delta V_{g} \sim 1 \mathrm{~V}$ seems present at $T=64 \mathrm{~K}$; we are unsure as to its origin.

[30] For a nonarmchair nanotube, the only difference would be $u \rightarrow u_{\text {armchair }} \cos (3 \theta)$, with $\theta=0$ for armchair, and varies up to $\theta=\pi / 6$ for zigzag tubes.

[31] B. L. Altshuler, A. G. Aronov, and D.E. Khmelnitskii, J. Phys. C 15, 7367 (1982).

[32] J. von Delft, F. Marquardt, R. A. Smith, and V. Ambegaokar, arXiv:cond-mat/0510557.

[33] A. Komnik and R. Egger, Phys. Rev. Lett. 80, 2881 (1998).

[34] H. W. C. Postma, M. de Jonge, Z. Yao, and C. Dekker, Phys. Rev. B 62, R10653 (2000).

[35] J. Jiang, J. Dong, and D. Y. Xing, Phys. Rev. Lett. 91, 056802 (2003).

[36] J. Cao et al., Phys. Rev. Lett. 93, 216803 (2004). 